JOURNAL OF

SYMPLECTIC GEOMETRY

Volume 3, Number 4, 749-767, 2005

\title{
COMPLEX AND KÄHLER STRUCTURES ON COMPACT SOLVMANIFOLDS
}

\author{
Keizo Hasegawa
}

\begin{abstract}
We discuss our recent results on the existence and classification problem of complex and Kähler structures on compact solvmanifolds. In particular, we determine in this paper all the complex surfaces which are diffeomorphic to compact solvmanifolds (and compact homogeneous manifolds in general).
\end{abstract}

\section{Introduction}

The purpose of this paper is to discuss our recent results on the existence and classification problem of complex and Kähler structures on compact solvmanifolds. In particular, we determine in this paper all the complex surfaces which are diffeomorphic to four-dimensional compact solvmanifolds (see Theorem 1); combined with many known results, this makes us determine the complete list of complex surfaces which are diffeomorphic to four-dimensional compact homogeneous manifolds (see Section 8).

A solvmanifold $M$ is a compact homogeneous space of solvable Lie group, that is, $M$ is a differentiable manifold on which a connected solvable Lie group $G$ acts transitively (and almost effectively). $M$ can be written as $D \backslash G$, where $G$ is a simply connected solvable Lie group and $D$ is a closed subgroup of $G$ (which includes no non-trivial connected normal subgroup of $G$ ). By complex structures (or Kähler structures) on solvmanifolds, we mean integrable almost complex structures (with compatible Kähler form) on $M$ which are not necessarily invariant by the canonical (right) action of $G$. A complex structure $J$ on $M$ is called left-invariant if it is induced from a left-invariant complex structure on $G$. It should be noted that complex structures on solvmanifolds may or may not be left-invariant; however, we shall see in this paper that four-dimensional solvmanifolds admit only left-invariant complex structures. We do not know any solvmanifolds 
(including tori) of higher dimension which admit non-left-invariant complex structures.

The classification of compact homogeneous Kähler manifolds is well known $[\mathbf{8}, \mathbf{2 6}]$; in particular, we know that the only compact homogeneous Kähler solvmanifolds are complex tori. On the other hand, we observed in the paper [20] that a four-dimensional solvmanifold admits a Kähler structure if and only if it is a complex torus or a hyperelliptic surface. The following theorem on four-dimensional solvmanifolds may be considered as a generalization of this result:

Theorem 1. A complex surface is diffeomorphic to a four-dimensional solvmanifold if and only if it is one of the following surfaces: Complex torus, Hyperelliptic surface, Inoue Surface of type $S^{0}$, Primary Kodaira surface, Secondary Kodaira surface, Inoue Surface of type $S^{ \pm}$. And every complex structure on each of these complex surfaces (considered as solvmanifolds) is left-invariant.

A hyperelliptic surface can be characterized as a finite quotient of a complex torus which is simultaneously a complex torus bundle over a complex torus. As a natural generalization of hyperelliptic surfaces to the higher dimension, we can define a class of Kählerian solvmanifolds (see Example 4). And as stated in the paper [20], we made a conjecture that any Kählerian solvmanifold must belong to this class of solvmanifolds. Recently, we have given a complete proof for this conjecture, applying a result of Arapura and Nori on polycyclic (solvable) Kähler groups [3] (see also [1]), together with our previous results on Kählerian nilmanifolds $[\mathbf{9}, \mathbf{1 8}]$ and Kählerian solvmanifolds [20] (see Section 7):

Theorem 2. [21]. A compact solvmanifold admits a Kähler structure if and only if it is a finite quotient of a complex torus which has a structure of a complex torus bundle over a complex torus. In particular, a compact solvmanifold of completely solvable type has a Kähler structure if and only if it is a complex torus.

The last part of the theorem was first conjectured by Benson and Gordon [10], which can be shown simultaneously in the proof of the theorem. For the definition of completely solvable type, we refer to Section 7.

In this paper, we provide many examples of solvmanifolds, including two class of four-dimensional solvmanifolds which admit no complex structures (see Section 4), and a class of six-dimensional pseudo-Kählerian solvmanifolds (of completely solvable type) which admit no complex structures but satisfy most of the known topological properties of compact Kähler manifolds (see Section 7). 


\section{Fundamental results on solvmanifolds}

We recall some fundamental results on solvmanifolds, most of which are found in [5]. Let $M$ be a solvmanifold of dimension $n$. We have the following basic results:

(1) $M$ is a fiber bundle over a torus with fiber a nilmanifold (which is called the Mostow fibration of $M$ ) [27]. In particular, we can represent the fundamental group $\Gamma$ of $M$ as an extension of a torsion-free nilpotent group $\Lambda$ of rank $n-k$ by a free abelian group of rank $k$, where $1 \leq k \leq n$ and $k=n$ if and only if $\Gamma$ is abelian:

$$
0 \longrightarrow \Lambda \longrightarrow \Gamma \longrightarrow \mathbf{Z}^{k} \longrightarrow 0 .
$$

(2) Conversely, any such abstract group $\Gamma$ (which is a polycyclic group) can be the fundamental group of some solvmanifold [35]. We call such a group $\Gamma$ a Wang group of rank $n$.

(3) It is also well known (due to Mostow [27]) that two solvmanifolds having isomorphic fundamental groups are diffeomorphic.

It is often useful to assume that $k=b_{1}$ (the first Betti number of $M$ ) in the group extension of (1), which is possible due to a result of Auslander and Szczarba [7] that a solvmanifold $M=D \backslash G$ has the canonical torus fibration over the torus $N D \backslash G$ of dimension $b_{1}$ with fiber a nilmanifold, where $N$ is the nilradical of $G$ (the maximal connected normal subgroup of $G$ ).

It should be noted that a solvmanifold $M$ is not necessarily of the form $\Gamma \backslash G$, where $G$ is a simply connected solvable Lie group with discrete subgroup $\Gamma$. However, it is known (due to Auslander [4]) that $M$ has a solvmanifold of the form $\Gamma^{\prime} \backslash G$ as a finite covering, where $\Gamma^{\prime}$ is a subgroup of $\Gamma$ with finite index. We know in general that two Wang groups are commensurable if and only if the corresponding two solvmanifolds has the same solvmanifold as a finite covering.

\section{Four-dimensional solvmanifolds with complex structures}

Let $\Gamma$ be the fundamental group of a four-dimensional solvmanifold $S$. Then we have

$$
0 \longrightarrow \Lambda \longrightarrow \Gamma \longrightarrow \mathbf{Z}^{k} \longrightarrow 0,
$$

where $\Lambda$ is a torsion-free nilpotent group of rank $4-k$.

For the classification of four-dimensional solvmanifolds (up to finite covering), it is sufficient to classify Wang groups $\Gamma$ as the group extensions of the above form and find a subgroup $\Gamma^{\prime}$ of finite index, which extends to a simply connected solvable Lie group $G$ such that $\Gamma^{\prime} \backslash G$ is a solvmanifold.

We define three types of Wang groups of rank 4 as follows: (Type I) $2 \leq k \leq 4$, (Type II) $k=1$ and $\Lambda$ is abelian, (Type III) $k=1$ and $\Lambda$ is 
non-abelian, where these three types are not mutually exclusive (as seen in Example 1).

Example 1. Let $\Lambda_{n}=\mathbf{Z}^{2} \rtimes \mathbf{Z}$, where the action $\phi: \mathbf{Z} \rightarrow \operatorname{Aut}\left(\mathbf{Z}^{2}\right)$ is defined by $\phi(1)=A_{n} \in \mathrm{GL}(2, \mathbf{Z})$,

$$
A_{n}=\left(\begin{array}{cc}
1 & n \\
0 & 1
\end{array}\right)
$$

Then $\Lambda_{n}$ is a nilpotent group of rank 3 and has a matrix expression:

$$
\Lambda_{n}=\left\{\left(\begin{array}{ccc}
1 & a & \frac{c}{n} \\
0 & 1 & b \\
0 & 0 & 1
\end{array}\right) \mid a, b, c \in \mathbf{Z}\right\}
$$

We see that $\Lambda_{n}$ can also be expressed as a non-split group extension:

$$
0 \longrightarrow \mathbf{Z} \longrightarrow \Lambda_{n} \longrightarrow \mathbf{Z}^{2} \longrightarrow 0
$$

where the action of $\mathbf{Z}^{2}$ on $\mathbf{Z}$ is trivial. It should be noted that a torsion-free nilpotent group of rank 3 is isomorphic to $\Lambda_{n}$ for some $n \in \mathbf{Z}$.

Let $\Gamma_{n}=\Lambda_{n} \times \mathbf{Z}$ be a nilpotent group of rank 4 . Then $\Gamma_{n}$ belongs to all of the types I, II, and III.

A four-dimensional solvmanifold $S$ is of type $I$ (II or $I I I$ ) if the fundamental group of $S$ is of type I (II or III, respectively). Concerning the four-dimensional solvmanifolds of type I, we have

Proposition 1. A four-dimensional solvmanifold $S$ is of type $I$ if and only if $S$ is a $T^{2}$ bundle over $T^{2}$.

Proof. We can easily see that Wang groups with $k=3$ or 4 can be expressed also as those with $k=2$. Hence, the solvmanifolds of type I are all $T^{2}$ bundles over $T^{2}$. Conversely, the fundamental group of a $T^{2}$ bundle over $T^{2}$ is clearly a Wang group. Since we know [30] that diffeomorphism types of $T^{2}$ bundles over $T^{2}$ are also determined uniquely by their fundamental groups, they are diffeomorphic to some solvmanifolds of dimension four.

It is known (due to Ue [32]) that a complex surface $S$ is diffeomorphic to a $T^{2}$ bundle over $T^{2}$ if and only if $S$ is a complex torus, Kodaira surface or hyperelliptic surface. The following result may be considered as a generalization of this result.

Theorem 1. A complex surface is diffeomorphic to a four-dimensional solvmanifold if and only if it is one of the following surfaces: Complex torus, Hyperelliptic surface, Inoue Surface of type $S^{0}$, Primary Kodaira surface, Secondary Kodaira surface, Inoue Surface of type $S^{ \pm}$. And every complex structure on each of these complex surfaces (considered as solvmanifolds) is left-invariant. 
The proof of Theorem 1 consists of three parts. The first part is to show that each of the complex surfaces (in the theorem) can be characterized as a solvmanifold of type II or III with canonical complex structure. The rest of this section is devoted to this part of the proof. The second part of the proof (the converse of the first part) is to show that a complex surface with diffeomorphism type of solvmanifold must be one of the complex surfaces in the theorem. We shall see this part of the proof in Section 6. For the proof of the last part of the theorem, we shall give in Section 5 the complete list of (left-invariant) complex structures on these complex surfaces as integrable almost complex structures on their corresponding solvable Lie algebras.

We now characterize each of the complex surfaces in the theorem as a solvable manifold of type II or III as follows.

[Type II] We have the following split group extension, where the action $\phi: \mathbf{Z} \rightarrow$ Aut $\mathbf{Z}^{3}$ is defined by $\phi(1) \in \mathrm{SL}(3, \mathbf{Z})$ :

$$
0 \longrightarrow \mathbf{Z}^{3} \longrightarrow \Gamma \longrightarrow \mathbf{Z} \longrightarrow 0 .
$$

(1) Complex tori. $\phi(1)=I$, and thus $\Gamma=\mathbf{Z}^{4}$.

(2) Hyperelliptic surfaces. $\phi(1)$ has a single root 1 and a double root -1 with linearly independent eigenvectors or non-real complex roots $\beta, \bar{\beta}$ with $|\beta|=1$.

(3) Inoue surfaces of type $S^{0} . \phi(1)$ has non-real complex roots $\alpha, \bar{\alpha}$ and a real root $c(c \neq 1)$ with $|\alpha|^{2} c=1$.

[Type III] The group extension is split and thus determined only by the action $\phi: \mathbf{Z} \rightarrow \operatorname{Aut}\left(\Lambda_{n}\right)$ :

$$
0 \longrightarrow \Lambda_{n} \longrightarrow \Gamma \longrightarrow \mathbf{Z} \longrightarrow 0,
$$

where $\Lambda_{n}$ is a nilpotent group of rank 3 as defined in Example 1.

An automorphism $\phi(1)$ induces the automorphism $\widetilde{\phi}(1)$ of the center $\mathbf{Z}$ of $\Lambda_{n}$ and the automorphism $\widehat{\phi}(1)$ of $\mathbf{Z}^{2}=\Lambda_{n} / \mathbf{Z}$.

(4) Primary Kodaira surfaces. $\phi(1)=\mathrm{Id}$, and thus $\Gamma=\Lambda_{n} \times \mathbf{Z}$.

(5) Secondary Kodaira surfaces. $\widetilde{\phi}(1)=\mathrm{Id}$, and $\widehat{\phi}(1)$ has a double root 1 with linearly independent eigenvectors or non-real complex roots $\alpha, \bar{\alpha}$ with $|\alpha|=1$.

(6) Inoue surfaces of type $S^{ \pm} . \widetilde{\phi}(1)= \pm \mathrm{Id}$, and $\widehat{\phi}(1)$ has two positive roots $a, b(a \neq 1)$ ) with $a b=1$ (two real roots $a, b$ with opposite sign $(|a| \neq 1)$ with $a b=-1$ respectively). Note that the Inoue surface of type $S^{-}$has that of type $S^{+}$as a double covering.

(1) Complex tori. An $n$-dimensional torus $T^{n}$ is a compact homogeneous space of the abelian Lie group $\mathbf{R}^{n}$ : that is, $T^{n}=\mathbf{Z}^{n} \backslash \mathbf{R}^{n}$, where $\mathbf{Z}^{n}$ is an abelian lattice of $\mathbf{R}^{n}$ which is spanned by some basis of $\mathbf{R}^{n}$ as a real vector 
space. For the case $n=2 m$, the standard complex structure $\mathbf{C}^{m}$ on $\mathbf{R}^{2 m}$ defines a complex structure on $T^{2 m}$. The complex manifold thus obtained is a complex torus.

It should be noted that $T^{n}(n \geq 3)$ can admit a structure of non-toral solvmanifold (see Section 7). On the other hand, it is unknown if all the complex structures on $T^{2 m}(m \geq 3)$ are the standard ones. This holds for $m=1,2$, since it does define a Riemann surface with $b_{1}=2$ (a complex surface with $b_{1}=4$, respectively), which is Kählerian, and its albanese map is biholomophic.

(2) Hyperelliptic surfaces. Let $\Gamma=\mathbf{Z}^{3} \rtimes \mathbf{Z}$, where the action $\phi: \mathbf{Z} \rightarrow \operatorname{Aut}\left(\mathbf{Z}^{3}\right)$ is defined by $\phi(1) \in \operatorname{SL}(3, \mathbf{Z})$. Assume that $\phi(1)$ has a single root 1 and a double root -1 , with linearly independent eigenvectors of -1 or non-real complex roots $\beta, \bar{\beta}$ with $|\beta|=1$.

For $A=\phi(1) \in \mathrm{SL}(3, \mathbf{Z})$ which satisfies our assumption, we can find a basis $\left\{u_{1}, u_{2}, u_{3}\right\}$ of $\mathbf{R}^{3}$ such that $A u_{1}=a u_{1}-b u_{2}, A u_{2}=b u_{1}+a u_{2}$, $A u_{3}=u_{3}$, where $a=-1$ and $b=0$, for the case that $A$ has a double root -1 and $a=\operatorname{Re} \beta$ and $b=\operatorname{Im} \beta$, for the case that $A$ has a non-real complex root $\beta$. Let $u_{i}=\left(u_{i 1}, u_{i 2}, u_{i 3}\right), i=1,2,3$. Then $\left\{v_{1}, v_{2}, v_{3}\right\}$, $v_{j}=\left(u_{1 j}, u_{2 j}, u_{3 j}\right), j=1,2,3$, defines an abelian lattice $\mathbf{Z}^{3}$ of $\mathbf{R}^{3}$ which is preserved by a rotation around a fixed axis. In particular, $\beta$ must be $e^{\sqrt{-1} \zeta}$ $\left(\zeta=\frac{2}{3} \pi, \frac{1}{2} \pi\right.$ or $\left.\frac{1}{3} \pi\right)$.

Furthermore, we may assume that $u_{3 j}=0, j=1,2$ and $A$ is of the form

$$
\left(\begin{array}{ccc}
a_{11} & a_{12} & 0 \\
a_{21} & a_{22} & 0 \\
p & q & 1
\end{array}\right),
$$

where $A^{\prime}=\left(a_{i j}\right) \in \mathrm{SL}(2, \mathbf{Z}), p, q \in \mathbf{Z}$. Since $A^{\prime}$ has the root -1 (with linearly independent eigenvectors) or $\beta$, we can assume that $A^{\prime}$ is of the form:

$$
\left(\begin{array}{cc}
-1 & 0 \\
0 & -1
\end{array}\right), \quad\left(\begin{array}{cc}
0 & 1 \\
-1 & -1
\end{array}\right), \quad\left(\begin{array}{cc}
0 & 1 \\
-1 & 0
\end{array}\right), \quad\left(\begin{array}{cc}
0 & 1 \\
-1 & 1
\end{array}\right),
$$

according to the root $e^{\sqrt{-1} \eta}$ of $A^{\prime}$, where $\eta=\pi, \frac{2}{3} \pi, \frac{1}{2} \pi$ or $\frac{1}{3} \pi$, respectively.

We now define a solvable Lie group $G=(\mathbf{C} \times \mathbf{R}) \rtimes \mathbf{R}$, where the action $\phi: \mathbf{R} \rightarrow \operatorname{Aut}(\mathbf{C} \times \mathbf{R})$ is defined by

$$
\phi(t)(z, s)=\left(\mathrm{e}^{\sqrt{-1} \eta t} z, s\right)
$$

which is a canonical extension of $\phi . \Gamma=\mathbf{Z}^{3} \rtimes \mathbf{Z}$ clearly defines a lattice of $G$. Since the action on the second factor $\mathbf{R}$ is trivial, the multiplication of $G$ is defined on $\mathbf{C}^{2}$ as follows:

$$
\left(w_{1}, w_{2}\right) \cdot\left(z_{1}, z_{2}\right)=\left(w_{1}+\mathrm{e}^{\sqrt{-1} \eta t} z_{1}, w_{2}+z_{2}\right),
$$


where $t=\operatorname{Re} w_{2}$. For each lattice $\Gamma$ of $G, S=\Gamma \backslash G$ with the canonical complex structure from $\mathbf{C}^{2}$ defines a complex surface which is, by definition, a hyperelliptic surface [11].

We can see that there exist seven isomorphism classes of lattices of $G$, which correspond to seven classes of hyperelliptic surfaces. For each $\eta$, take a lattice $\mathbf{Z}^{3}$ of $\mathbf{R}^{3}$ spanned by $\left\{v_{1}, v_{2}, v_{3}\right\}$ for $A$ with $p=q=0$. Then we can get a lattice $\mathbf{Z}^{3}$ spanned by $\left\{v_{1}, v_{2}, v_{3}^{\prime}\right\}$ for $A$ with arbitrary $(p, q) \in \mathbf{Z}^{2}$, by changing $v_{3}$ into $v_{3}^{\prime}=s v_{1}+t v_{2}+v_{3}$, where $s, t \in \mathbf{Q}$ with $0 \leq s, t<1$, and $\Gamma=\mathbf{Z}^{3} \rtimes \mathbf{Z}$ defines a lattice of the solvable Lie group $G$. By elementary calculation, we obtain the following seven isomorphism classes of lattices: besides four trivial cases with $(p, q)=(0,0)$ and $(s, t)=(0,0)$ for $\eta=$ $\pi, \frac{2}{3} \pi, \frac{1}{2} \pi$, and $\frac{1}{3} \pi$, we have three other cases with $(p, q)=(1,0) ;(\mathrm{i})(s, t)=$ $\left(\frac{1}{2}, 0\right)$ for $\eta=\pi$, (ii) $(s, t)=\left(\frac{1}{3}, \frac{1}{3}\right)$ for $\eta=\frac{2}{3} \pi$, (iii) $(s, t)=\left(\frac{1}{2}, \frac{1}{2}\right)$ for $\eta=\frac{1}{2} \pi$.

(3) Inoue surfaces of type $S^{0}$. Let $\Gamma=\mathbf{Z}^{3} \rtimes \mathbf{Z}$, where the action $\phi: \mathbf{Z} \rightarrow$ $\operatorname{Aut}\left(\mathbf{Z}^{3}\right)$ is defined by $\phi(1) \in \mathrm{SL}(3, \mathbf{Z})$. Assume that $\phi(1)$ has complex roots $\alpha, \bar{\alpha}$ and a real root $c(c \neq 1)$ with $|\alpha|^{2} c=1$.

Let $\left(\alpha_{1}, \alpha_{2}, \alpha_{3}\right) \in \mathbf{C}^{3}$ be the eigenvector of $\alpha$ and $\left(c_{1}, c_{2}, c_{3}\right) \in \mathbf{R}^{3}$ the eigenvector of $c$. The set of vectors $\left\{\left(\alpha_{i}, c_{i}\right) \in \mathbf{C} \times \mathbf{R} \mid i=1,2,3\right\}$ are linearly independent over $\mathbf{R}$ and defines a lattice $\mathbf{Z}^{3}$ of $\mathbf{C} \times \mathbf{R}$. Then $\Gamma=\mathbf{Z}^{3} \rtimes \mathbf{Z}$ can be extended to a solvable Lie group $G=(\mathbf{C} \times \mathbf{R}) \rtimes \mathbf{R}$, where the action $\bar{\phi}: \mathbf{R} \rightarrow \operatorname{Aut}(\mathbf{C} \times \mathbf{R})$ is defined by $\bar{\phi}(t):(z, s) \rightarrow\left(\alpha^{t} z, c^{t} s\right)$.

Taking a coordinate change $\mathbf{R} \rightarrow \mathbf{R}_{+}$defined by $t \rightarrow e^{\log c t}$ and regarding $\mathbf{R} \times \mathbf{R}_{+}$as $\mathbf{H}$ (the upper half plain), $M=\Gamma \backslash G$ can be considered as $\mathbf{C} \times \mathbf{H} / \Gamma^{\prime}$, where $\Gamma^{\prime}$ is a group of automorphisms generated by $g_{0}$ and $g_{i}$, $i=1,2,3$, which correspond to the canonical generators of $\Gamma$. To be more precise, we see

$$
g_{0}:\left(z_{1}, z_{2}\right) \rightarrow\left(\alpha z_{1}, c z_{2}\right), \quad g_{i}:\left(z_{1}, z_{2}\right) \rightarrow\left(z_{1}+\alpha_{i}, z_{2}+c_{i}\right), \quad i=1,2,3 .
$$

$S=\mathbf{C} \times \mathbf{H} / \Gamma^{\prime}$ is, by definition, an Inoue surface of type $S^{0}[\mathbf{2 3}]$.

(4) Primary Kodaira surfaces. Let $\Gamma_{n}=\Lambda_{n} \times \mathbf{Z}$ (a nilpotent group of rank 4). $\Gamma_{n}$ can be extended to the nilpotent Lie group $G=N \times \mathbf{R}$, where

$$
N=\left\{\left(\begin{array}{lll}
1 & x & s \\
0 & 1 & y \\
0 & 0 & 1
\end{array}\right) \mid x, y, s \in \mathbf{R}\right\} .
$$

Taking the coordinate change $\Phi$ from $N \times \mathbf{R}$ to $\mathbf{R}^{4}$ :

$$
\Phi:((x, y, s), t) \longrightarrow\left(x, y, 2 s-x y, 2 t+\frac{1}{2}\left(x^{2}+y^{2}\right)\right),
$$

and regarding $\mathbf{R}^{4}$ as $\mathbf{C}^{2}$, the group operation on $G$ can be expressed as

$$
\left(w_{1}, w_{2}\right) \cdot\left(z_{1}, z_{2}\right)=\left(w_{1}+z_{1}, w_{2}-\sqrt{-1} \bar{w}_{1} z_{1}+z_{2}\right) .
$$


Let $\Gamma_{n}^{\prime}$ be the corresponding group of affine transformations on $\mathbf{C}^{2}$, then $S=\mathbf{C}^{2} / \Gamma_{n}^{\prime}$ is, by definition, a Primary Kodaira surface $[\mathbf{2 4}]$.

(5) Secondary Kodaira surfaces. Let $\Gamma_{n}=\Lambda_{n} \rtimes \mathbf{Z}$, where the action $\phi: \mathbf{Z} \rightarrow$ $\operatorname{Aut}\left(\Lambda_{n}\right)$ satisfies the condition that the induced automorphism $\widetilde{\phi}(1)$ of $\mathbf{Z}$ is trivial, that is, $\widetilde{\phi}(1)=\mathrm{Id}$, and the induced automorphism $\widehat{\phi}(1)$ of $\mathbf{Z}^{2}$ has a double root -1 with linearly independent eigenvectors, or non-real complex roots, $\alpha, \bar{\alpha}(\alpha \neq \bar{\alpha})$ with $|\alpha|=1$.

We shall see that $\Gamma_{n}$ can be extended to a solvable Lie group $G=N \rtimes \mathbf{R}$. As we have seen in (2), $\alpha$ must be $e^{i \eta}, \eta=\pi, \frac{2}{3} \pi, \frac{1}{2} \pi$ or $\frac{1}{3} \pi$, and there exists a basis $\left\{u_{1}^{\prime}, u_{2}^{\prime}\right\}$ of $\mathbf{R}^{2}$ such that $A u_{1}^{\prime}=a u_{1}^{\prime}-b u_{2}^{\prime}, A u_{2}^{\prime}=b u_{1}^{\prime}+a u_{2}^{\prime}$, where $A=\widehat{\phi}(1), a=\operatorname{Re} \alpha, b=\operatorname{Im} \alpha$ and $u_{1}^{\prime}=\left(u_{11}, u_{12}\right), u_{2}^{\prime}=\left(u_{21}, u_{22}\right)$. The abelian lattice $\mathbf{Z}^{2}$ of $\mathbf{R}^{2}$ spanned by $\left\{v_{1}^{\prime}, v_{2}^{\prime}\right\}$, where $v_{1}^{\prime}=\left(u_{11}, u_{21}\right), v_{2}^{\prime}=$ $\left(u_{12}, u_{22}\right)$, is preserved by the automorphism $\psi^{\prime}:(x, y) \rightarrow(a x-b y, b x+a y)$ of $\mathbf{R}^{2}$. We can extend $\psi^{\prime}$ to an automorphism of $N$ of the form:

$$
\psi:\left(x, y, \frac{z}{n}\right) \longrightarrow\left(a x-b y, b x+a y, \frac{z}{n}+h(x, y)\right),
$$

where $h(x, y)=\frac{1}{2} b\left(a x^{2}-a y^{2}-2 b x y\right)$. We can extend the lattice $\mathbf{Z}^{2}$ spanned by $\left\{v_{1}^{\prime}, v_{2}^{\prime}\right\}$ to a lattice $\Lambda_{n}$ spanned by $\left\{v_{1}, v_{2}, v_{3}\right\}, v_{1}=\left(u_{11}, u_{21}, u_{31}\right), v_{2}=$ $\left(u_{12}, u_{22}, u_{32}\right), v_{3}=\left(0,0, u_{33}\right)$, for suitable $u_{31}, u_{32}, u_{33}$, so that $\Lambda_{n}$ is preserved by $\psi$. We now define a solvable Lie group $G$ by extending the action $\phi(m)=\psi^{m}, m \in \mathbf{Z}$ to $\psi(t), t \in \mathbf{R}$, replacing $a$ with $\cos \eta t$ and $b$ with $\sin \eta t$. It is clear that $\Gamma_{n}=\Lambda_{n} \rtimes \mathbf{Z}$ defines a lattice of $G$. If we take the new coordinate as in (4), the automorphism $\psi$ is expressed as

$$
\left(z_{1}, z_{2}\right) \longrightarrow\left(\zeta z_{1}, z_{2}\right)
$$

for $\zeta \in \mathbf{C},|\zeta|=1$. It follows that the above automorphism is holomorphic with respect to the complex structure defined in (3). We see that $S_{n}=\Gamma_{n} \backslash G$ is a finite quotient of a primary Kodaira surface; and $S_{n}$ with the above complex structure is, by definition, a secondary Kodaira surface $[\mathbf{1 1}, \mathbf{1 3}]$.

(6) Inoue surfaces of type $S^{ \pm}$. Let $\Gamma_{n}=\Lambda_{n} \rtimes \mathbf{Z}$, where the action $\phi: \mathbf{Z} \rightarrow$ $\operatorname{Aut}\left(\Lambda_{n}\right)$ satisfies the condition that for the induced action $\widetilde{\phi}: \mathbf{Z} \rightarrow \operatorname{Aut}(\mathbf{Z})$, $\widetilde{\phi}(1)=\mathrm{Id}$, and for the induced action $\widehat{\phi}: \mathbf{Z} \rightarrow \operatorname{Aut}\left(\mathbf{Z}^{2}\right), \widehat{\phi}(1)=\left(n_{i j}\right) \in$ $\mathrm{SL}(2, \mathbf{Z})$ has two positive real roots $a, b$ with $a b=1$.

Let $\left(a_{1}, a_{2}\right),\left(b_{1}, b_{2}\right) \in \mathbf{R}^{2}$ be eigenvectors of $a, b$, respectively. Let $G=$ $N \rtimes \mathbf{R}$ be a solvable Lie group, where the action $\bar{\phi}: \mathbf{R} \rightarrow \operatorname{Aut}(N)$ is defined by

$$
\bar{\phi}(t):\left(\begin{array}{ccc}
1 & x & z \\
0 & 1 & y \\
0 & 0 & 1
\end{array}\right) \longrightarrow\left(\begin{array}{ccc}
1 & a^{t} x & z \\
0 & 1 & b^{t} y \\
0 & 0 & 1
\end{array}\right)
$$


which is a canonical extension of $\phi$. In order to define a lattice $\Lambda_{n}$ which is preserved by $\bar{\phi}$, we take $g_{1}, g_{2}, g_{3} \in N$ as

$$
g_{1}=\left(\begin{array}{ccc}
1 & a_{1} & c_{1} \\
0 & 1 & b_{1} \\
0 & 0 & 1
\end{array}\right), \quad g_{2}=\left(\begin{array}{ccc}
1 & a_{2} & c_{2} \\
0 & 1 & b_{2} \\
0 & 0 & 1
\end{array}\right), \quad g_{3}=\left(\begin{array}{ccc}
1 & 0 & c_{3} \\
0 & 1 & 0 \\
0 & 0 & 1
\end{array}\right),
$$

where $c_{1}, c_{2}, c_{3}$ are to be determined, satisfying the following conditions:

(1) $\left[g_{1}, g_{2}\right]=g_{3}^{n}$

(2) $\bar{\phi}(1)\left(g_{1}\right)=g_{1}^{n_{11}} g_{2}^{n_{12}} g_{3}^{k}, \bar{\phi}(1)\left(g_{2}\right)=g_{1}^{n_{21}} g_{2}^{n_{22}} g_{3}^{l}$, where $k, l \in \mathbf{Z}$.

If we take $g_{0} \in N \rtimes \mathbf{R}$ as

$$
g_{0}=\left(\left(\begin{array}{lll}
1 & 0 & p \\
0 & 1 & 0 \\
0 & 0 & 1
\end{array}\right), 1\right), \quad p \in \mathbf{R}
$$

then $\left\{g_{0}, g_{1}, g_{2}, g_{3}\right\}$ defines a lattice $\Gamma_{n}$ of $G$, and $S_{n}=\Gamma_{n} \backslash G$ is a solvmanifold.

Now, we define a diffeomorphism $\Psi: G=N \rtimes \mathbf{R} \longrightarrow \mathbf{R}^{3} \times \mathbf{R}_{+}$, for an arbitrary $\gamma=p+q \sqrt{-1} \in \mathbf{C}$ and $\sigma=\log b$, by

$$
\Psi:\left(\left(\begin{array}{ccc}
1 & y & x \\
0 & 1 & s \\
0 & 0 & 1
\end{array}\right), t\right) \longrightarrow\left(x, e^{\sigma t} y+q t, s, e^{\sigma t}\right) .
$$

Then considering $\mathbf{R}^{3} \times \mathbf{R}_{+}$as $\mathbf{C} \times \mathbf{H}, g_{0}, g_{1}, g_{2}, g_{3}$ are corresponding to the following holomorphic automorphisms of $\mathbf{C} \times \mathbf{H}$,

$$
\begin{gathered}
g_{0}:\left(z_{1}, z_{2}\right) \longrightarrow\left(z_{1}+\gamma, b z_{2}\right), \\
g_{i}:\left(z_{1}, z_{2}\right) \longrightarrow\left(z_{1}+a_{i} z_{2}+c_{i}, z_{2}+b_{i}\right),
\end{gathered}
$$

where $i=1,2,3$ and $a_{3}=b_{3}=0 . S_{n}$ with the above complex structure is, by definition, an Inoue surface of type $S^{+}[\mathbf{2 3}]$.

An Inoue surface of type $S^{-}$is defined similarly as the case where the action $\phi: \mathbf{Z} \rightarrow \operatorname{Aut}\left(\Lambda_{n}\right)$ satisfies the condition that $\widetilde{\phi}(1)=-\mathrm{Id}$, and $\widehat{\phi}(1)$ has a positive and a negative real root. It is clear that an Inoue surface of type $S^{-}$has $S^{+}$with $\gamma=0$ as its double covering surface.

\section{Examples}

We give in this section three other classes of four-dimensional (orientable) solvmanifolds which (as a consequence of Theorem 1) admit no complex structures.

Example 2. Let $\Gamma$ be the Wang group of type II defined by the following split group extension, where the action $\phi: \mathbf{Z} \rightarrow$ Aut $^{3}$ is defined by $\phi(1) \in$ $\mathrm{SL}(3, \mathbf{Z})$ :

$$
0 \longrightarrow \mathbf{Z}^{3} \longrightarrow \Gamma \longrightarrow \mathbf{Z} \longrightarrow 0
$$


(1) Suppose that $\phi(1)$ has three distinct positive real roots $a_{1}, a_{2}, a_{3}$, then there exist linearly independent eigenvectors $u_{1}, u_{2}, u_{3}$ of $a_{1}, a_{2}, a_{3}$ respectively. Let $u_{i}=\left(u_{i 1}, u_{i 2}, u_{i 3}\right), i=1,2,3$. Then we have an abelian lattice $\mathbf{Z}^{3}$ of $\mathbf{R}^{3}$ defined by $\left\{v_{1}, v_{2}, v_{3}\right\}, v_{j}=\left(u_{1 j}, u_{2 j}, u_{3 j}\right)$, $j=1,2,3$. We define a solvable Lie group $G=\mathbf{R}^{3} \rtimes \mathbf{R}$, where the action $\phi: \mathbf{R} \rightarrow \operatorname{Aut}\left(\mathbf{R}^{3}\right)$ is defined by

$$
\phi(t)(x, y, z)=\left(e^{t \log a_{1}} x, e^{t \log a_{2}} y, e^{t \log a_{3}} z\right),
$$

which is a canonical extension of $\phi$. Then $\Gamma=\mathbf{Z}^{3} \rtimes \mathbf{Z}$ is a lattice of $G$, and $S=\Gamma \backslash G$ is a solvmanifold. We can see that $S$ is a $T^{2}$-bundle over $T^{2}$ with $b_{1}=2$ for the case where one of the roots is 1 and a $T^{3}$ bundle over $T^{1}$ with $b_{1}=1$ for the case where none of the roots is 1 .

(2) Suppose that $\phi(1)$ has a triple root 1, then taking a suitable basis $\left\{u_{1}, u_{2}, u_{3}\right\}$ of $\mathbf{R}^{3}, \phi(1)$ is expressed in either of the following forms:

$$
\left(\begin{array}{lll}
1 & 1 & \frac{1}{2} \\
0 & 1 & 1 \\
0 & 0 & 1
\end{array}\right), \quad\left(\begin{array}{lll}
1 & 1 & 0 \\
0 & 1 & 0 \\
0 & 0 & 1
\end{array}\right) .
$$

Let $G=\mathbf{R}^{3} \rtimes \mathbf{R}$, where the action $\bar{\phi}: \mathbf{R} \rightarrow \operatorname{Aut}\left(\mathbf{R}^{3}\right)$ is defined by

$$
\bar{\phi}(t)=\exp t\left(\begin{array}{ccc}
0 & 1 & 0 \\
0 & 0 & 1 \\
0 & 0 & 0
\end{array}\right)=\left(\begin{array}{ccc}
1 & t & \frac{1}{2} t^{2} \\
0 & 1 & t \\
0 & 0 & 1
\end{array}\right)
$$

for the former case, and

$$
\bar{\phi}(t)=\exp t\left(\begin{array}{lll}
0 & 1 & 0 \\
0 & 0 & 0 \\
0 & 0 & 0
\end{array}\right)=\left(\begin{array}{lll}
1 & t & 0 \\
0 & 1 & 0 \\
0 & 0 & 1
\end{array}\right)
$$

for the latter case. Then, as defined in case (1), $\left\{v_{0}, v_{1}, v_{2}, v_{3}\right\}$ defines a lattice $\Gamma$ of $G$, and $S=\Gamma \backslash G$ is a nilmanifold. We can see that $S$ is a nilmanifold with $b_{1}=2$ for the former case (which admits no complex structures) and a nilmanifold with $b_{1}=3$ for the latter case (which is a primary Kodaira surface).

Remark 1. We can show that, up to finite covering, there exist nine classes of four-dimensional (orientable) solvmanifolds: six classes of complex surfaces in Section 3 and three classes of solvmanifolds in Example 2, which admit no complex structures.

Example 3. Let $\Gamma_{n}$ be a Wang group expressed as the extension:

$$
0 \longrightarrow \mathbf{Z}^{2} \longrightarrow \Gamma_{n} \longrightarrow \mathbf{Z}^{2} \longrightarrow 0
$$


where the action $\phi: \mathbf{Z}^{2} \rightarrow \operatorname{Aut}\left(\mathbf{Z}^{2}\right)$ is defined by $\phi\left(e_{1}\right), \phi\left(e_{2}\right), e_{1}=(1,0)$, $e_{2}=(0,1)$. Suppose that $\phi\left(e_{1}\right)=-\mathrm{I}$ and $\phi\left(e_{2}\right)$ is of the form

$$
\left(\begin{array}{ll}
1 & n \\
0 & 1
\end{array}\right)
$$

Then we can express $\Gamma_{n}$ also as $\Lambda_{n} \rtimes \mathbf{Z}$, where $\Lambda_{n}$ is the nilpotent group of rank 3 as defined in Example 1, and the action $\phi: \mathbf{Z} \rightarrow \operatorname{Aut}\left(\Lambda_{n}\right)$ is defined by $\phi(1)=\tau \in \operatorname{Aut}\left(\Lambda_{n}\right)$,

$$
\tau:\left(\begin{array}{ccc}
1 & x & \frac{z}{n} \\
0 & 1 & y \\
0 & 0 & 1
\end{array}\right) \longrightarrow\left(\begin{array}{ccc}
1 & x & \frac{-z}{n} \\
0 & 1 & -y \\
0 & 0 & 1
\end{array}\right) .
$$

Let $G=N \times \mathbf{R}$ be a nilpotent Lie group, where $N$ is the nilpotent Lie group obtained by the real completion of $\Lambda_{n}$ as defined in (4). $\Gamma_{n}$ acts freely as a group of automorphisms on $G$, and $S_{n}=G / \Gamma_{n}$ is a solvmanifold with $b_{1}=2$. $S_{n}$ has the nilmanifold $\left(\Lambda_{n} \times \mathbf{Z}\right) \backslash G$ as a double covering, with the covering transformation group $\mathbf{Z}_{2}$ generated by $\tau$.

We show that $S_{n}$ is parallelizable, that is, $S_{n}$ admits a field of linear frame (consisting of four linearly independent vector fields). We have three linearly independent left-invariant vector fields on $N$ :

$$
X_{1}=\frac{\partial}{\partial x}, \quad X_{2}=\frac{\partial}{\partial y}+n x \frac{\partial}{\partial z}, \quad X_{3}=\frac{\partial}{\partial z} .
$$

We define vector fields on $G$ invariant by $\tau$ : $\widetilde{X}_{1}=X_{1}, \widetilde{X}_{4}=\partial / \partial t$ and

$$
\widetilde{X}_{2}=\cos (\pi t) X_{2}+\sin (\pi t) X_{3}, \quad \widetilde{X}_{3}=-\sin (\pi t) X_{2}+\cos (\pi t) X_{3},
$$

which are linearly independent and invariant by $\Gamma_{n}$.

It should be noted that $S_{n}$ can be expressed as $D_{n} \backslash \bar{G}$, where $\bar{G}$ is the extension of $G$ with $y, z \in \mathbf{C}$ and the action $\bar{\phi}$ defined by

$$
\bar{\phi}(t)(x, y, z)=\left(x, e^{\sqrt{-1} \pi t} y, e^{\sqrt{-1} \pi t} z\right),
$$

and $D_{n}=\Gamma_{n} H$, where $H$ is the closed subgroup of $\bar{G}$ with $x=0, y=$ $\sqrt{-1} y_{2}, z=\sqrt{-1} z_{2}, y_{2}, z_{2} \in \mathbf{R}$.

Remark 2. As noted in the paper [6], all of the four-dimensional solvmanifolds are parallelizable. This is trivial for the case where $S$ is of the form $\Gamma \backslash G$ (where $\Gamma$ is a simply connected solvable Lie group with lattice $\Gamma$ ). For the case where $S$ is not of the form $\Gamma \backslash G$, we can see that, as shown in Example 3, $S$ always admits a field of linear frame.

\section{Complex structures on solvable Lie algebras}

A four-dimensional solvmanifold can be written (up to finite covering) as $\Gamma \backslash G$, where $\Gamma$ is a lattice of a simply connected solvable Lie group $G$. In 
this section, we express complex structures of the complex surfaces in the last section as those induced from left-invariant complex structures on $G$ (i.e., left-invariant complex structures), by defining integrable almost complex structures $J$ on the Lie algebra $\mathfrak{g}$ of $G$. In the following list, we express the Lie algebra $\mathfrak{g}$ of $G$ as having a basis $\left\{X_{1}, X_{2}, X_{3}, X_{4}\right\}$ with the bracket multiplication specified for each complex surface. Except for (6), the almost complex structure $J$ is defined by

$$
J X_{1}=X_{2}, \quad J X_{2}=-X_{1}, \quad J X_{3}=X_{4}, \quad J X_{4}=-X_{3},
$$

for which the Nijenhuis tensor $N_{J}\left(X_{i}, X_{j}\right)=\left[J X_{i}, J X_{j}\right]-J\left[J X_{i}, X_{j}\right]-$ $J\left[X_{i}, J X_{j}\right]-\left[X_{i}, X_{j}\right], 1 \leq i<j \leq 4$, vanishes.

(1) Complex tori. $\left[X_{i}, X_{j}\right]=0(1 \leq i<j \leq 4)$.

(2) Hyperelliptic surfaces. $\left[X_{4}, X_{1}\right]=-X_{2},\left[X_{4}, X_{2}\right]=X_{1}$, and all other brackets vanish.

(3) Inoue surfaces of type $S^{0}$. $\left[X_{4}, X_{1}\right]=a X_{1}-b X_{2},\left[X_{4}, X_{2}\right]=b X_{1}+a X_{2}$, $\left[X_{4}, X_{3}\right]=-2 a X_{3}$, and all other brackets vanish, where $a, b(\neq 0) \in \mathbf{R}$.

(4) Primary Kodaira surfaces. $\left[X_{1}, X_{2}\right]=-X_{3}$, and all other brackets vanish.

(5) Secondary Kodaira surfaces. $\left[X_{1}, X_{2}\right]=-X_{3},\left[X_{4}, X_{1}\right]=-X_{2}$, $\left[X_{4}, X_{2}\right]=X_{1}$, and all other brackets vanish.

(6) Inoue surfaces of type $S^{+}$and $S^{-}$. $\left[X_{2}, X_{3}\right]=-X_{1},\left[X_{4}, X_{2}\right]=X_{2}$, $\left[X_{4}, X_{3}\right]=-X_{3}$, and all other brackets vanish. The almost complex structure $J$ is defined by

$$
J X_{1}=X_{2}, \quad J X_{2}=-X_{1}, \quad J X_{3}=X_{4}-q X_{2}, \quad J X_{4}=-X_{3}-q X_{1},
$$

for which the Nijenhuis tensor $N_{\mathrm{J}}$ vanishes.

\section{Proof of Theorem 1}

We have seen in Sections 3 and 5 that each of the complex surfaces in Theorem 1 can be characterized as a four-dimensional solvmanifold with canonical left-invariant complex structure. In this section, we shall complete the proof of Theorem 1, showing the converse that a complex surface with diffeomorphism of solvmanifold must be one of the complex surfaces in the theorem.

We denote by $S$ a complex surface with diffeomorphism type of solvmanifold. We first remark that since $S$ is parallelizable, the Euler number $c_{2}$ of $S$ vanishes, and the fundamental group of $S$ is abelian if and only if $S$ is a four-dimensional torus [27]. Let $\kappa(S)$ be the Kodaira dimension of $S$. The classification of complex surfaces with $c_{2}=0$ is divided into three cases: $\kappa(S)=-\infty, 0,1[\mathbf{1 1}]$. In the case where $\kappa(S)=-\infty, S$ is a surface of class $\mathrm{VII}_{0}$ or ruled surface of genus 1 . The latter surface cannot be diffeomorphic to a solvmanifold since the fundamental group of ruled surface of genus 1 is 
$\mathbf{Z}^{2}$ (which is abelian). According to the well-known theorem of Bogomolov (proved by Li et al. [15]), we know that a complex surface of class $\mathrm{VII}_{0}$ with $b_{1}=1$ and $c_{2}=0$ is an Inoue surface or a Hopf surface. Since the fundamental group of the Hopf surface is of the form $H \rtimes \mathbf{Z}$, where $H$ is a finite unitary group (including the trivial case), it cannot be the fundamental group of solvmanifold. Hence, $S$ must be an Inoue surface. In the case where $\kappa(S)=0, S$ is a complex torus, hyperelliptic surface or Kodaira surface (primary or secondary Kodaira surface).

In the case where $\kappa(S)=1, S$ is a (properly) elliptic surface (which is minimal since $c_{2}=0$ ). Let us first recall some terminologies and fundamental results concerning topology of elliptic surfaces in general. An elliptic surface is a complex surface $S$ together with an elliptic fibration $f: S \rightarrow B$, where $B$ is a curve, such that a general fiber $f^{-1}(t), t \in B$ (except finite points $\left.t_{1}, t_{2}, \ldots, t_{k}\right)$ is an elliptic curve. The base curve $B$ is regarded as a two-dimensional orbifold with multiple points $t_{i}$ with multiplicity $m_{i}$, where $m_{i}(i \geq 2)$ is the multiplicity of the fiber $f^{-1}\left(t_{i}\right)(i=1,2, \ldots, k)$. An elliptic surface $S$ is of the type hyperbolic, flat (Euclidean), spherical or bad, according as the orbifold $B$ is of that type. The Euler number $e^{\text {orb }}(B)$ of $B$ is by definition $e(B)-\sum_{i=1}^{k}\left(1-\frac{1}{m_{i}}\right)$, where $e(B)$ is the Euler number of $B$ as topological space. We know that $B$ is hyperbolic, flat or spherical according as $e^{\operatorname{orb}}(B)$ is negative, 0 or positive. In the case $c_{2}=0$, we can see $[33,34]$ that an elliptic surface $S$ is hyperbolic, flat or spherical according as the Kodaira dimension $\kappa(S)$ is 1,0 or $-\infty$. We now continue our proof for the case $\kappa(S)=1$. By the above argument, $S$ is a minimal elliptic surface of hyperbolic type. We show that the fundamental group of $S$ is not solvable, and thus $S$ cannot be diffeomorphic to a solvmanifold. We have the following presentation of $\pi_{1}(S)$ as a short exact sequence [13]:

$$
0 \longrightarrow \mathbf{Z}^{2} \longrightarrow \pi_{1}(S) \longrightarrow \pi_{1}^{\text {orb }}(B) \longrightarrow 0,
$$

where $\pi_{1}^{\text {orb }}(B)$ is the fundamental group as two-dimensional orbifold.

Since $\pi_{1}^{\text {orb }}(B)$ is a discrete subgroup of $\operatorname{PSL}(2, \mathbf{R})$, it contains a torsionfree subgroup $\Gamma$ of finite index, such that $\Gamma$ is the fundamental group of a finite orbifold covering $\widetilde{B}$ of $B$, which is a closed surface of genus $g \geq 2$ (or a Riemann surface of hyperbolic type) $[\mathbf{2 8}]$. We know that $\Gamma$ is represented as a group with generator $\left\{a_{1}, a_{2}, \ldots, a_{g}, b_{1}, b_{2}, \ldots, b_{g}\right\}$ and relation $\prod_{i=1}^{g}\left[a_{i}, b_{i}\right]=1$, which is not solvable for $g \geq 2[\mathbf{1 2}]$. It follows that $\pi_{1}(S)$ cannot be solvable since the quotient groups and subgroups of a solvable group must be solvable. This completes the proof of Theorem 1.

\section{Solvmanifolds with Kähler structures}

Let $M$ be a solvmanifold of the form $\Gamma \backslash G$, where $\Gamma$ is a lattice of a simply connected solvable Lie group $G$. $M$ is of completely solvable type, if the 
adjoint representation of the Lie algebra $\mathfrak{g}$ of $G$ has only real eigenvalues and of rigid type (or of type $(R)$ ), in the sense of Auslander, if the adjoint representation of $\mathfrak{g}$ has only pure imaginary (including 0 ) eigenvalues. It is clear that $M$ is both of completely solvable and of rigid type if and only if $\mathfrak{g}$ is nilpotent, that is, $M$ is a nilmanifold. As we have seen in Sections 4 and 5, a hyperelliptic surface can be characterized as a solvmanifold of rigid type with canonical complex structure. We define a natural generalization of hyperelliptic surfaces as in the following example.

Example 4. Let $G=\mathbf{C}^{l} \rtimes \mathbf{R}^{2 k}$ with the action $\phi: \mathbf{R}^{2 k} \rightarrow \operatorname{Aut}\left(\mathbf{C}^{l}\right)$ defined by

$$
\phi\left(\bar{t}_{i}\right)\left(z_{1}, z_{2}, \ldots, z_{l}\right)=\left(e^{\sqrt{-1} \eta_{1}^{i} t_{i}} z_{1}, e^{\sqrt{-1} \eta_{2}^{i} t_{i}} z_{2}, \ldots, e^{\sqrt{-1} \eta_{l}^{i} t_{i}} z_{l}\right),
$$

where $\bar{t}_{i}=t_{i} e_{i}\left(e_{i}\right.$ : the $i$ th unit vector in $\left.\mathbf{R}^{2 k}\right)$ and $e^{\sqrt{-1}} \eta_{j}^{i}$ is the $s_{i}$ th root of unity, $i=1, \ldots, 2 k, j=1, \ldots, l$. If an abelian lattice $\mathbf{Z}^{2 l}$ of $\mathbf{C}^{l}$ is preserved by the action $\phi$ on $\mathbf{Z}^{2 k}$, then $M=\Gamma \backslash G$ defines a solvmanifold of rigid type, where $\Gamma=\mathbf{Z}^{2 l} \rtimes \mathbf{Z}^{2 k}$ is a lattice of $G$. In fact, the Lie algebra $\mathfrak{g}$ of $G$ is the following:

$$
\mathfrak{g}=\left\{X_{1}, X_{2}, \ldots, X_{2 l}, X_{2 l+1}, \ldots, X_{2 l+2 k}\right\},
$$

where the bracket multiplications are defined by

$$
\left[X_{2 l+2 i}, X_{2 j-1}\right]=-X_{2 j}, \quad\left[X_{2 l+2 i}, X_{2 j}\right]=X_{2 j-1}
$$

for $i=1, \ldots, k, j=1, \ldots, l$, and all other brackets vanish. The canonical left-invariant complex structure is defined by

$$
\begin{gathered}
J X_{2 j-1}=X_{2 j}, \quad J X_{2 j}=-X_{2 j-1}, \quad J X_{2 l+2 i-1}=X_{2 l+2 i}, \\
J X_{2 l+2 i}=-X_{2 l+2 i-1}
\end{gathered}
$$

for $i=1, \ldots, k, j=1, \ldots, l$.

Remark 3. Let $G=\mathbf{C}^{l} \rtimes \mathbf{R}^{2 k}$ with the action $\phi: \mathbf{R}^{2 k} \rightarrow \operatorname{Aut}\left(\mathbf{C}^{l}\right)$ defined by

$$
\phi\left(\bar{t}_{i}\right)\left(z_{1}, z_{2}, \ldots, z_{l}\right)=\left(e^{2 \pi \sqrt{-1} t_{i}} z_{1}, e^{2 \pi \sqrt{-1} t_{i}} z_{2}, \ldots, e^{2 \pi \sqrt{-1} t_{i}} z_{l}\right),
$$

where $\bar{t}_{i}=t_{i} e_{i}\left(e_{i}\right.$ : the $i$-the unit vector in $\left.\mathbf{R}^{2 k}\right), i=1, \ldots, 2 k$. Then $\mathbf{Z}^{2 n} \backslash G$ is a solvmanifold diffeomorphic to a torus $T^{2 n}(n=k+l)$.

It is easily seen that $M$ (in Example 4) is a finite quotient of a complex torus and has a structure of a complex torus bundle over a complex torus. The following theorem asserts that any solvmanifolds which admit Kähler structures must belong to the class of Kählerian solvmanifolds defined in Example 4. Note that a solvmanifold of completely solvable type does not belong to this class unless it is a complex torus:

Theorem 2 [21]. A compact solvmanifold admits a Kähler structure if and only if it is a finite quotient of a complex torus which has a structure of a complex torus bundle over a complex torus. In particular, a compact 
solvmanifold of completely solvable type has a Kähler structure if and only if it is a complex torus.

The proof of the theorem is based on a result of Aparura and Nori [3] that a polycyclic Kähler group has a nilpotent subgroup of finite index, together with our previous results: a result of the papers $[\mathbf{9}, \mathbf{1 8}]$ that the only Kählerian nilmanifold is a complex torus and a partial result on Kählerian solvmanifolds in the paper [20]. For the details of the proof and the related topics, we refer to the paper $[\mathbf{2 1}]$.

We give an example of a six-dimensional solvmanifold which admits a psuedo-Kähler structures but no Kähler structures.

Example $5[\mathbf{2}]$. Let $\Lambda=\mathbf{Z}^{4} \rtimes \mathbf{Z}$, with the action $\phi: \mathbf{Z} \rightarrow \operatorname{Aut}\left(\mathbf{Z}^{4}\right)$ defined by $\phi(1)=A \oplus A \in \operatorname{Aut}\left(\mathbf{Z}^{4}\right)$, where $A \in \operatorname{SL}(2, \mathbf{Z})$ has two positive eigenvalues $a_{1}, a_{2}\left(a_{1} \neq 1, a_{1} a_{2}=1\right)$. Let $u=\left(u_{1}, u_{2}\right), v=\left(v_{1}, v_{2}\right)$ be the eigenvectors of $a_{1}, a_{2}$, respectively. We have a basis $\left\{\left(u_{1}, v_{1}, 0,0\right),\left(u_{2}, v_{2}, 0,0\right),\left(0,0, u_{1}, v_{1}\right)\right.$, $\left.\left(0,0, u_{2}, v_{2}\right)\right\}$ of $\mathbf{R}^{4}$, which defines an abelian lattice $\mathbf{Z}^{4}$ of $\mathbf{R}^{4}$. We define a solvable Lie group $H=\mathbf{R}^{4} \rtimes \mathbf{R}$ with the action $\bar{\phi}: \mathbf{R} \rightarrow \operatorname{Aut}\left(\mathbf{R}^{4}\right)$ defined by

$$
\bar{\phi}(t)\left(x_{1}, x_{2}, y_{1}, y_{2}\right)=\left(e^{t \log a_{1}} x_{1}, e^{t \log a_{2}} x_{2}, e^{t \log a_{1}} y_{1}, e^{t \log a_{2}} y_{2}\right),
$$

which is a canonical extension of $\phi$. Then, $\Gamma=\Lambda \times \mathbf{Z}$ is a lattice of a solvable Lie group $G=H \times \mathbf{R}$, and $M=\Gamma \backslash G$ is a six-dimensional solvmanifold.

The Lie algebra $\mathfrak{g}$ of $G$ is expressed as having a basis $\left\{X_{1}, X_{2}, Y_{1}, Y_{2}, Z, W\right\}$ with the bracket multiplication:

$$
\left[X_{1}, Z\right]=X_{1}, \quad\left[X_{2}, Z\right]=-X_{2}, \quad\left[Y_{1}, Z\right]=Y_{1}, \quad\left[Y_{2}, Z\right]=-Y_{2},
$$

and all other brackets vanishing. Let $\alpha_{1}, \alpha_{2}, \beta_{1}, \beta_{2}, \gamma, \eta$ be the corresponding Maurer-Cartan forms (left-invariant 1-forms), then we have

$d \alpha_{1}=\gamma \wedge \alpha_{1}, \quad d \alpha_{2}=-\gamma \wedge \alpha_{2}, \quad d \beta_{1}=\gamma \wedge \beta_{1}, \quad d \beta_{2}=-\gamma \wedge \beta_{2}, \quad d \gamma=d \eta=0$.

Let $\omega=\alpha_{1} \wedge \alpha_{2}+\beta_{1} \wedge \beta_{2}+\gamma \wedge \eta$, then $\omega$ defines a left-invariant symplectic form on $M$. A complex structure $J$ on $\mathfrak{g}$ is defined by

$J X_{1}=Y_{1}, \quad J Y_{1}=-X_{1}, \quad J X_{2}=Y_{2}, \quad J Y_{2}=-X_{2}, \quad J Z=W, \quad J W=-Z$.

It is easy to check that the Nijenhuis tensor vanishes for $J$ and that the pair $(\omega, J)$ defines a psuedo-Kähler structure on $M$. Since $G$ is of completely solvable type, we can apply a result of Hattori $[\mathbf{2 2}]$ that the De Rham cohomology ring of $M$ is isomorphic to the cohomology ring of $\mathfrak{g}$. It is now not hard to check that with respect to $(\omega, J)$ the following "Kähler conditions" hold for $M$ (while $M$ never admits Kähler structures due to Theorem 1): (1) the Hard Lefschetz condition, (2) the Betti number $b_{2 k-1}$ are even, (3) the De Rham complex is formal and (4) the Fröhlicher spectral sequence degenerates at $E_{1}$. For more details, we refer to the papers $[\mathbf{2}, \mathbf{3}]$. 


\section{Four-dimensional compact homogeneous manifolds with complex structures}

In this section, we determine all the complex surfaces which are diffeomorphic to four-dimensional compact homogeneous manifolds.

It is known (due to Gorbatsevich $[\mathbf{1 6}, \mathbf{1 7}]$ ) that a four-dimensional compact homogeneous manifold is diffeomorphic to one of the following types: (1) $\prod S^{k_{i}}$ (up to finite quotient), where $k_{i} \geq 1$ with $\sum k_{i}=4$; (2) $\mathbf{C P}^{2}$; (3) solvmanifold; (4) $S^{1} \times \Gamma \backslash \widetilde{S L}_{2}(\mathbf{R})$, where $\widetilde{\mathrm{SL}}_{2}(\mathbf{R})$ is the universal covering of $\mathrm{SL}_{2}(\mathbf{R})$ and $\Gamma$ is a lattice of $\widetilde{S L}_{2}(\mathbf{R})$. We can determine, based on the above result, the complete list of complex surfaces with diffeomorphism type of four-dimensional compact homogeneous manifolds:

\begin{tabular}{|c|c|c|c|}
\hline$S$ & $b_{1}$ & Complex structure & $\kappa$ \\
\hline$S^{2} \times T^{2}$ & 2 & Ruled surface of genus 1 & \\
\hline$S^{1} \times \mathbf{z}_{m} S^{3} / H$ & 1 & Hopf surface & \\
\hline$S^{2} \times S^{2}$ & 0 & Hirzebruch surface of even type & $-\alpha$ \\
\hline $\mathrm{CP}^{2}$ & 0 & Complex projective space & \\
\hline \multirow{5}{*}{ Solvmanifold \{} & 1 & Inoue surface & \\
\hline & 4 & Complex torus & \multirow{4}{*}{0} \\
\hline & 3 & Primary kodaira surface & \\
\hline & 2 & Hyperelliptic surface & \\
\hline & 1 & Secondary kodaira surface & \\
\hline$S 1 \times \Gamma \backslash \widehat{S L}_{2}(\mathbf{R})$ & Odd & Properly elliptic surface & 1 \\
\hline
\end{tabular}

where $\kappa$ is the Kodaira dimension of $S$ and $\mathrm{H}$ is a finite subgroup of $\mathrm{SU}(2)$ acting freely on $S^{3}$.

It is well known (due to Borel and Serre) that $S^{4}$ has almost no complex structure. For the case of $S^{2} \times T^{2}$, it is known (due to Suwa [29]) that a complex surface is diffeomorphic to a $S^{2}$-bundle over $T^{2}$ if and only if it is a ruled surface of genus 1 . We can see this also from the recent result (due to Friedman and Qin [14]) that the Kodaira dimension of complex algebraic surface is invariant up to diffeomorphism. To be more precise, there exist two diffeomorphism types of ruled surfaces of genus 1: the trivial one and the non-trivial one (which correspond to two diffeomorphism types of $S^{2}$ bundles over $T^{2}$ ), and the latter is not of homogeneous space form [29].

For the case of $S^{1} \times S^{3}$, Kodaira [25] showed that a complex surface diffeomorphic to a finite quotient of $S^{1} \times S^{3}$ is a Hopf surface. Generally, a Hopf surface is diffeomorphic to a fiber bundle over $S^{1}$ with fiber $S^{3} / \mathrm{U}$, defined by the action $\rho: \pi_{1}\left(S^{1}\right) \rightarrow N_{\mathrm{U}(2)}(\mathrm{U})$ with $\rho(1)$ being cyclic of order $m$, where $\mathrm{U}$ is a finite subgroup of $\mathrm{U}(2)$ acting freely on $S^{3}$ : that is, $S=$ $S^{1} \times \mathbf{z}_{m} S^{3} / U[\mathbf{1 9}]$. We can see that a Hopf surface is of homogeneous space 
form if and only if $U$ is a finite subgroup of $\mathrm{SU}(2)$. Let $G=\mathrm{SU}(2) \times S^{1}$, which is a compact Lie group structure on $S^{3} \times S^{1}$. Take a finite subgroup $\Delta=H \rtimes \mathbf{Z}_{m}$ of $G$, where $H$ is a finite subgroup of $\mathrm{SU}(2), \mathbf{Z}_{m}$ is a finite cyclic subgroup of $G$ generated by $c$ :

$$
c=(\tau, \xi), \quad \tau=\left(\begin{array}{cc}
\xi^{-1} & 0 \\
0 & \xi
\end{array}\right), \quad \xi^{m}=1,
$$

and $\tau$ belongs to $N_{\mathrm{SU}(2)}(H) . S$ is a fiber bundle over $S^{1}$ with fiber $S^{3} / H$, which has a canonical complex structure, defining a Hopf surface. It should be noted that if $\tau$ does not belong to $H$ and $m \geq 2$, then $S$ is a non-trivial bundle. Conversely, given a Hopf surface $S$ with fiber $S^{3} / H$, defined by the action $\rho$, we can assume that $\rho(1)$ is a diagonal matrix, all of which entries are $m$-th roots of 1 [19]. Then we can see that $\rho(1)$, which belongs to $N_{\mathrm{U}(2)}(H)$, actually belongs to $N_{\mathrm{SU}(2)}(H)$. Hence, $S$ is diffeomorphic to the one constructed above.

For the case of $S^{2} \times S^{2}$, it was shown (due to Qin [31]) that a complex surface diffeomorphic to $S^{2} \times S^{2}$ must be a Hirzebruch surface of even type, which is by definition a ruled surface of genus 0 with diffeomorphism type $S^{2} \times S^{2}$. As is well known, there exist two diffeomorphism types of ruled surfaces of genus 0: $S^{2} \times S^{2}$ and $\mathbf{C P}^{2} \# \overline{\mathbf{C P}^{2}}$ (which correspond to two diffeomorphism types of $S^{2}$-bundles over $S^{2}$ ). A Hirzebruch surface of odd type is the surface of the latter type. We can see that no non-trivial finite quotient of $S^{2} \times S^{2}$ has a complex structure. It is well known (due to Yau) that $\mathbf{C P}^{2}$ can have only the standard complex structure. We have studied in detail the case of solvmanifolds in this paper. The complex surfaces with diffeomorphism type of solvmanifolds are Inoue surfaces for $\kappa=-\infty$ and all of those with $c_{2}=0$ for $\kappa=0$. For the case of $S^{1} \times \Gamma \backslash \widetilde{\mathrm{SL}_{2}}(\mathbf{R})$, Wall [33] showed that it admits a canonical complex structure, which defines a properly elliptic surface with $b_{1}=$ odd and $c_{2}=0$; and conversely any such surface with no singular fibers is diffeomorphic to $S^{1} \times \Gamma \backslash \widetilde{S L}_{2}(\mathbf{R})$ for some lattice $\Gamma$.

\section{References}

[1] J. Amorós, M. Burger, K. Corlette, D. Kotschick and D. Toledo, Fundamental groups of compact Kähler manifolds, Mathematical Surveys and Monographs, 44, American Mathematical Society.

[2] L.C. De Andŕes, M. Fernández, M. De Leon and J.J. Mencía, Some six-dimensional compact symplectic and complex solvmanifolds, Rendicondi di Math., Series VII, 12 (1992), 59-67.

[3] D. Arapura and M. Nori, Solvable fundamental groups of algebraic varieties and Kähler manifolds, Compositio Math. 116(2) (1999), 173-188.

[4] L. Auslander, Discrete uniform subgroups of solvable Lie groups, Trans. Am. Math. Soc., 99(2) (1961), 398-402. 
[5] L. Auslander, An exposition of the structure of solvmanifolds, I, II, Bull. Am. Math. Soc., 79(2) (1973), 227-261, 262-285.

[6] L. Auslander and R.H. Szczarba, Characteristic classes of compact Solvmanifolds, Ann. Math. 76 (1962), 1-8.

[7] L. Auslander and H. Szczarba, Vector bundles over noncompact solvmanifolds, Am. J. Math. 97 (1975), 260-281.

[8] A. Borel, Kählerian coset spaces of semisimple Lie groups, Nat. Acad. Sci. USA, 40 (1954), 1147-1151.

[9] C. Benson and C.S. Gordon, Kähler and symplectic structures on nilmanifolds, Topology 27 (1988), 755-782.

[10] C. Benson and C.S. Gordon, Kähler structures on compact solvmanifolds, Proc. Am. Math. Soc. 108 (1990), 971-980.

[11] W. Barth, C. Peters and Van de Ven, Compact complex surfaces, Ergebnisse der Mathematik und ihrer Grenzgebiete, 4, Springer-Verlag, 1984.

[12] K.T. Chen, Algebras of iterated path integrals and fundamental groups, Trans. Am. Math. Soc. 156 (1971), 359-379.

[13] R. Friedman and J.W. Morgan, Smooth four-manifolds and complex surfaces, Ergebnisse der Mathematik und ihrer Grenzgebiete, 27, Springer-Verlag, 1994.

[14] R. Friedman and Z.B. Qin, On complex surfaces diffeomorphic to rational surfaces, Invent. Math. 120 (1995), 81-117.

[15] J. Li, S.T. Yau and F. Zheng, A simple proof of Bogomolov's theorem on class VII surfaces with $b_{2}=0$, Illinois J. Math. 34 (1990), 217-220.

[16] V.V. Gorbatsevich, On the classification of four-dimensional compact homogeneous spaces, Usp. Mat. Nauk., (Russian) 32(2) (1977), 207-208.

[17] V.V. Gorbatsevich and A.L. Onishchik, Lie transformation groups, Encyclopaedia of Mathematical Sciences, 20, Lie Groups and Lie Algebras I, Springer-Verlag, 1994.

[18] K. Hasegawa, Minimal models of nilmanifolds, Proc. Am. Math. Soc. 106 (1989), 65-71.

[19] K. Hasegawa, Deformations and diffeomorphism types of generalized Hopf manifolds, Illinois J. Math. 37 (1993), 643-651.

[20] K. Hasegawa, A class of compact Kählerian solvmanifolds and a general conjecture, Geometriae Dedicata 78 (1999), 253-258.

[21] K. Hasegawa, A note on compact solvmanifolds with Kähler structures, Osaka J. Math. 43(1) (2006).

[22] A. Hattori, Spectral sequence in the De Rham cohomology of fiber bundles, J. Fac. Sci. Univ. Tokyo 8 (1960), 289-331.

[23] M. Inoue, On surfaces of class VII , Inventiones Math. 24 (1974), 269-310.

[24] K. Kodaira, On the structure of compact complex analytic surfaces, I. Am. J. Math. 86 (1964), 751-798; On the structure of compact complex analytic surfaces, II, Am. J. Math. 88 (1966), 682-721.

[25] K. Kodaira, Complex structures on $S^{3} \times S^{1}$, Proc. Nat. Acad. Sci. 55 (1966), 240-243.

[26] Y. Matsushima, Sur les espaces homogènes Kählériens d'un groupe de Lie réductif, Nagoya Math. J. 11 (1957), 53-60.

[27] G.D. Mostow, Factor spaces of solvable Lie groups, Ann. Math. 60 (1954), 1-27. 
[28] P. Scott, The geometries of 3-manifolds, Bull. Lond. Math. Soc. 15 (1983), 401-487.

[29] T. Suwa, Ruled surfaces of genus, 1, J. Math. Soc. Japan 21 (1969), 291-311.

[30] K. Sakamoto and S. Fukuhara, Classification of $T^{2}$ bundles over $T^{2}$, Tokyo J. Math. 6 (1983), 311-327.

[31] Z.B. Qin, Complex structure on certain differential 4-manifolds, Topology 32 (1993), $551-566$.

[32] M. Ue, Geometric 4-manifolds in the sense of Thurston and Seifert 4-manifolds, I, J. Math. Soc. Japan 42 (1990), 511-540.

[33] C.T.C. Wall, Geometric structures on compact complex analytic surfaces, Topology 25 (1986), 119-153.

[34] C.T.C. Wall, Geometries and geometric structures in real dimension 4 and complex dimension 2, Lecture Notes Math. 1167 (1986), 118-153.

[35] H.C. Wang, Discrete subgroups of solvable Lie groups, I, Ann. Math. 64 (1956), 1-19.

Department of Mathematics,

Faculty of Education and Human Sciences,

Nimgata University,

NiIgATA, JAPAN

E-mail address: hasegawa@ed.niigata-u.ac.jp

Received 03/03/2005, revised 09/02/2005. The author would like to thank V.V. Gorbatsevich for valuable comments; in particular, the results in Section 8 were obtained while communicating with him by e-mail. The author appreciates very much the referee's valuable comments which lead to many improvements in the paper. Finally, the author would like to express a sincere gratitude to A. Tralle for research correspondence and invitation to the conference. 
